Fall 11-1-2012

\title{
Initial experience with Imacor hTEE-guided management of patients following transplant and mechanical circulatory support.
}

\author{
Christopher Kang \\ Hitoshi Hirose \\ Thomas Jefferson University \\ Harold Hasting
}

Follow this and additional works at: https://jdc.jefferson.edu/cbfp

Part of the Surgery Commons

Let us know how access to this document benefits you

\section{Recommended Citation}

Kang, Christopher; Hirose, Hitoshi; Hasting, Harold; and , "Initial experience with Imacor hTEEguided management of patients following transplant and mechanical circulatory support." (2012). Department of Cancer Biology Faculty Papers. Paper 31.

https://jdc.jefferson.edu/cbfp/31

This Article is brought to you for free and open access by the Jefferson Digital Commons. The Jefferson Digital Commons is a service of Thomas Jefferson University's Center for Teaching and Learning (CTL). The Commons is a showcase for Jefferson books and journals, peer-reviewed scholarly publications, unique historical collections from the University archives, and teaching tools. The Jefferson Digital Commons allows researchers and interested readers anywhere in the world to learn about and keep up to date with Jefferson scholarship. This article has been accepted for inclusion in Department of Cancer Biology Faculty Papers by an authorized administrator of the Jefferson Digital Commons. For more information, please contact: JeffersonDigitalCommons@jefferson.edu. 


\title{
As submitted to:
}

\author{
ICU Director
}

And later published as:

Initial Experience with Imacor hTEE-guided management of patients following Transplant and Mechanical Circulatory

\section{Support.}

\section{August 22, 2012, \\ doi:10.1177/1944451612456703}

\begin{abstract}
We reviewed an initial experience using a miniaturized transesophageal echocardiography (TEE) probe (ImaCor, Garden City, NY) with 3 patients in whom Imacor hTEE intervention was used as a point of care device to manage extracorporeal membrane oxygenation cannula placement, cardiac hemodynamics, and post-op cardiac pathophysiology. The management of transplant or mechanical circulatory support (MCS) patients is especially challenging: transplanted hearts pose unique pathophysiological challenges, and MCS significantly alters pressure-volume-flow relationships. Real-time "hemodynamic" TEE (hTEE) can help provide effective management by direct visualization of cardiac filling and function and help guide hemodynamic management. In our initial experience, hTEE can provide point-of-care management following transplant or MCS. (Word count of abstract: 105)
\end{abstract}


Keywords: ECMO, TEE, mechanical circulatory support, hemodynamics

\section{Conflict of Interest}

$\mathrm{XXXX}$ is an employee and stockholder in ImaCor. Other authors has no conflict of

interest. This research received no specific grant from any funding agency in the public, commercial, or not-for-profit sectors. 


\section{Introduction}

Fluid and hemodynamic management, fundamental in critical care, becomes significantly more challenging in transplant patients and patients with mechanical circulatory support. Transplanted hearts pose unique pathophysiological challenges ${ }^{1,2}$, and mechanical support significantly alters pressure-volume-flow relationships. These alterations can further limit the utility of standard pressure measurements, both central venous pressure and pulmonary artery pressure. Central venous pressure has been shown to have a poor relationship with volume status. ${ }^{3}$ The Swan-Ganz catheter provides indirect measurements that can be difficult to interpret; moreover altered pressurevolume-flow relationships. Transesophageal echocardiography (TEE) directly visualizes the heart, allowing accurate assessment of cardiac function and volume status with other parameters. ${ }^{4}$ Cardiac anesthesiologists rely on TEE images to make hemodynamic assessments and interventions in the cardiac operating room. TEE would appear to be the ideal tool for hemodynamic assessment and management of critically ill patients ${ }^{5}$, provided that availability and the need for trained personnel and anesthesia can be overcome. An editorial called for a TEE probe which could remain indwelling 48 hours in critically ill patients. ${ }^{6}$

To address these issues, a miniaturized TEE probe (hTEE probe, ImaCor, Garden City, NY) with a diameter of $5.5 \mathrm{~mm}$ was developed to provide hemodynamic management and assessment in critical care to aid in assessment of volume status and cardiac function with other parameters. The hTEE probe was cleared by the FDA to remain indwelling up to 72 hours. The hTEE probe has $15 \mathrm{~cm}$ of penetration at 6.67 
$\mathrm{MHz}$ (B-mode) and can obtain the images of left and right ventricular function and volume status. $^{7}$

\section{Methods}

We reviewed our initial experience with 3 patients in whom the Imacor hTEE system was used as a point-of-care device to manage extracorporeal membrane oxygenation (ECMO) cannula placement, cardiac hemodynamics, and post-op cardiac pathophysiology. This study was approved by an institutional review board at Thomas Jefferson University Hospital.

\section{Results}

Case 1: A 55-year-old female with a history of dilated cardiomyopathy, presented for a heart transplantation evaluation. She quickly decompensated into multiple organ failure despite significant inotropic support. It was decided that emergent Veno-arterial ECMO placement for biventricular support was necessary (Figure 1A). Over 3 weeks of VA ECMO support, the patient was deemed not a candidate for heart transplantation. Although the patient remained eligible for a left ventricular (LV) assist device, right ventricular $(\mathrm{RV})$ function had not been evaluated prior to decompensation.

A weaning protocol was initiated with hTEE for direct visualization of cardiac function to assess RV function. Over four hours, ECMO flows were decreased, and volume and dobutamine were administered. hTEE showed that the right heart was adequate to tolerate a bridge to LVAD (Figure 1B). The patient was successfully weaned off ECMO and bridged to an LVAD as predicted.

Case 2: A 47-year-old male with a Heartmate II LVAD (Thoratec, Pleasanton, CA) underwent orthotropic heart transplantation. Postoperatively, the patient required 
escalating vasopressors despite good cardiac function determined by a Swan-Ganz catheter using both thermodilution and mixed venous saturation. Twelve hours later, the Swan-Ganz catheter showed tamponade physiology with equalization of pulmonary artery diastolic pressure $(26 \mathrm{~mm} \mathrm{Hg})$ and central venous pressure $(25 \mathrm{~mm} \mathrm{Hg})$ with decreased cardiac index $\left(2.0 \mathrm{~L} / \mathrm{min} / \mathrm{cm}^{2}\right)$. An hTEE probe was placed to assess to evaluate LV and RV cardiac function; no tamponade was seen (Figure 2A). Real time imaging with continuous hTEE demonstrated deteriorating biventricular function to near cardiac standstill (Figure 2B). Based on hTEE visualization, central ECMO was established as a rescue support.

Case 3: A 58-year-old female with an acute anterior ST-elevation myocardial infarction underwent a percutaneous catheter intervention to the left anterior descending artery. During this intervention, she developed a complete AV block and cardiogenic shock. Her hemodynamics did not improve despite multiple inotropes and an intra-aortic balloon pump placement. An Impella (Abiomed, Danvers, MA) was emergently placed for circulatory support. However, it was complicated with a retroperitoneal hematoma and dislodgement of the Impella device. The patient developed severe pulmonary edema and was transferred to our institution for VA ECMO placement. As soon as VA ECMO was established at a flow of 4 to 4.5 liters/min, her hemodynamics significantly improved and stabilized. Approximately 6 hours later, her ECMO flows suddenly dropped to 2 liters/min. There were no changes of CVP (10 mm Hg) or PA pressure (32/12 mm Hg). A fluid challenge did not improve the flow. The hTEE probe was inserted and revealed a large right atrial thrombus interfering with the inflow to the venous cannula (Figure $3 \mathrm{~A}$ ). The ECMO cannula was adjusted bedside and heparin administration was initiated. The 
atrial clot was monitored real time using the hTEE probe (Figure 3B) and the clot dissolved over 24 hours.

\section{Discussion}

These 3 cases illustrate how Imacor hTEE intervention was used as a point of care device to manage extracorporeal membrane oxygenation (ECMO) cannula placement, cardiac hemodynamics, and post-op cardiac pathophysiology. The management of transplant or mechanical circulatory support patients is especially challenging: transplanted hearts pose unique pathophysiological challenges, and mechanical circulatory support significantly alters pressure-volume-flow relationships. Real-time "hemodynamic" TEE (hTEE) can help provide effective management by direct visualization of cardiac filling and function and help guide hemodynamic management.

Weaning from ECMO requires recovery of either RV and/or LV cardiac function. Although the first patient had persistent LV dysfunction, RV function recovered after 3 weeks of ECMO support. This allowed the patient to receive a HeartMateII LVAD. ECMO weaning requires ongoing observation of cardiac function through major circulatory changes which are visualized by real time continuous hTEE. A ECMO weaning protocol includes a gradual decrease of ECMO flows from full (usually 4-5 liter/min) to minimum flow (1.5-2 litter/min) over 4 to 6 hours, with appropriate increased anticoagulation to avoid clot formation, volume to fill the heart, and inotropic support such as dobutamine or milrinone to optimize ventricular contractility along with careful hemodynamic monitoring for arrhythmias, oxygenation, and blood pressure. ${ }^{8}$ Adequate filling pressures or adequate cardiac output do not necessarily imply adequate RV or LV function for patient on ECMO. Placement of a Swan-Ganz catheter (SGC) 
may be difficult with a venous cannula in a decompressed right atrium. A central mixed venous gas analysis would also be useful to evaluate the circulation and function of the heart; however, the lag between the sampling the blood and results may makes ongoing real-time adjustments difficult. The hTEE probe provides real time images of the heart, allowing the intensivist to determine if the preload is appropriate and cardiac function is adequate and intervene rapidly if necessary. If the heart distends during weaning, one can immediately return to the full ECMO support to avoid the further cardiac damage over-distension. If ventricular recovery is questionable, hTEE probe can be left in the patient for continuous monitoring of the cardiac function, potentially saving the cost of taking the patient to the operating room.

Primary biventricular failure post-transplantation, as seen in Case 2, may be related to poor myocardial preservation of the donor, prolonged ischemic time, residual pulmonary hypertension, and decreased compliance of the lungs post cardiopulmonary bypass. ${ }^{1,2}$ The HeartMate II LVAD in Case 2 decompressed the left ventricle, artificially lowering pulmonary artery pressure (Pap) and masking residual high pulmonary vascular resistance. Heart transplant patient with prolonged ischemic time and elevated recipient pulmonary vascular resistance often develop RV failure, which can progress to biventricular failure. $^{2}$

RV dysfunction post heart transplant may cause SGC parameters to mimic cardiac tamponade. A SGC can also reproduce unreliable readings with diastolic dysfunction due to the presence of a hematoma and myocardial edema. Cardiac tamponade should be readily diagnosed by TEE (including hTEE); however, due to our limited prior experience with hTEE, the patient was explored surgically and no tamponade was found. 
If we followed our hTEE findings, we would have avoided surgical exploration, a significant cost saving. ${ }^{10}$ This patient's RV failure progressed to biventricular failure and was retrospectively found to be caused by graft rejection. As seen here, indirect hemodynamic monitoring may be inadequate to diagnose RV or graft failure posttransplant. Moreover, in the presence of escalating vasopressor support secondary to systemic vasoplegia syndrome, normal pressure may not yield normal perfusion. This lack of normal perfusion was the apparent cause of near ventricular standstill detected by hTEE, a condition which if not managed by central ECMO might otherwise have led to cardiac arrest.

Case 3 illustrates an acute emergency in ECMO patients requiring rapid diagnosis and management, namely lack of or sharply reduced flow. Sharply reduced flow is usually related to the volume status of the patient, and resolved by administering volume. Sharply reduced flow shortly after ECMO placement may also be due to cannula malposition. In case 3 a thrombus in the right atrium occluded the cannula, a rare phenomenon. The hTEE probe allows us to visualization the thrombus, and its separation from the cannula following manipulation, at which time ECMO flow returned to normal. The hTEE probe was left indwelling until stabilization of ECMO flows (48 hours) to evaluate the right atrial thrombus. We were able to avoid unnecessary fluid boluses, which could be harmful in the presence of pulmonary edema.

\section{Conclusion}

We found hTEE helped guide point-of-care management of patients posttransplant or with mechanical circulatory support accurately and acutely. ${ }^{10}$ Although further study is needed, hTEE appeared easy to use by intensivists on demand and 
yielded significant management changes, potentially avoiding re-operation with concomitant mortality and morbidity ${ }^{11}$ as well as cost. 


\section{References}

1. Costanzo MR, Dipchand A, Starling R, et al; International Society of Heart and Lung Transplantation Guidelines. The International Society of Heart and Lung Transplantation Guidelines for the care of heart transplant recipients. J Heart Lung Transplant. 2010; 29:914-56.

2. Stobierska-Dzierzek B, Awad H, Michler RE. The evolving management of acute right-sided heart failure in cardiac transplant recipients. J Am Coll Cardiol. 2001; 38:923-31.

3. Marik PE, Baram M, Vahid B. Does central venous pressure predict fluid responsiveness? A systematic review of the literature and the tale of seven mares. Chest. $208 ; 134: 172-8$.

4. Poelaert JI, Schüpfer G. Hemodynamic monitoring utilizing transesophageal echocardiography: the relationships among pressure, flow, and function. Chest. 2005; 127: 379-90.

5. Vincent JL, Rhodes A, Perel A, et al. Clinical review: Update on hemodynamic monitoring--a consensus of 16. Crit Care. 2011; 15:229.

6. Greenhalgh DL, Patrick MR. Perioperative transoesophageal echocardiography: past, present \& future. Anaesthesia. 2012; 67:343-6.

7. Hastings, HM. Transesophageal echocardiography-guided hemodynamic assessment and management. ICU Director. 2012; 3:38-41.

8. Aissaoui N, Luyt CE, Leprince P, et al. Predictors of successful extracorporeal membrane oxygenation (ECMO) weaning after assistance for refractory cardiogenic shock. Intensive Care Med. 2011; 37:1738-45. 
9. Speir A, KasirajanV. Additive Costs of Postoperative Complications for Isolated Coronary Artery Bypass Grafting Patients in Virginia. Ann Thorac Surg. 2009;88:40-6. 10. Vieillard-Baron A, Charron C, Chergui K, Peyrouset O, Jardin F. Bedside echocardiographic evaluation of hemodynamics in sepsis: is a qualitative evaluation sufficient? Intensive Care Med. 2006; 32:1547-52.

11. Ranucci M, Bozzetti G. Surgical Reexploration After Cardiac Operations: Why a Worse Outcome? Ann Thorac Surg. 2008; 86: 1557-62. 


\section{Figure Legends}

Figure 1. A. Biventricular failure at the time of ECMO insertion. B. RV function improved with 3 weeks of ECMO support although the LV remained dilated. LV: left ventricle; RV: right ventricle. Line in the RV indicates $\mathrm{RV}$ dimension. Please note the $\mathrm{RV}$ dimension is smaller in Figure $1 \mathrm{~B}$ than $1 \mathrm{~A}$, indicating the recovery of RV function.

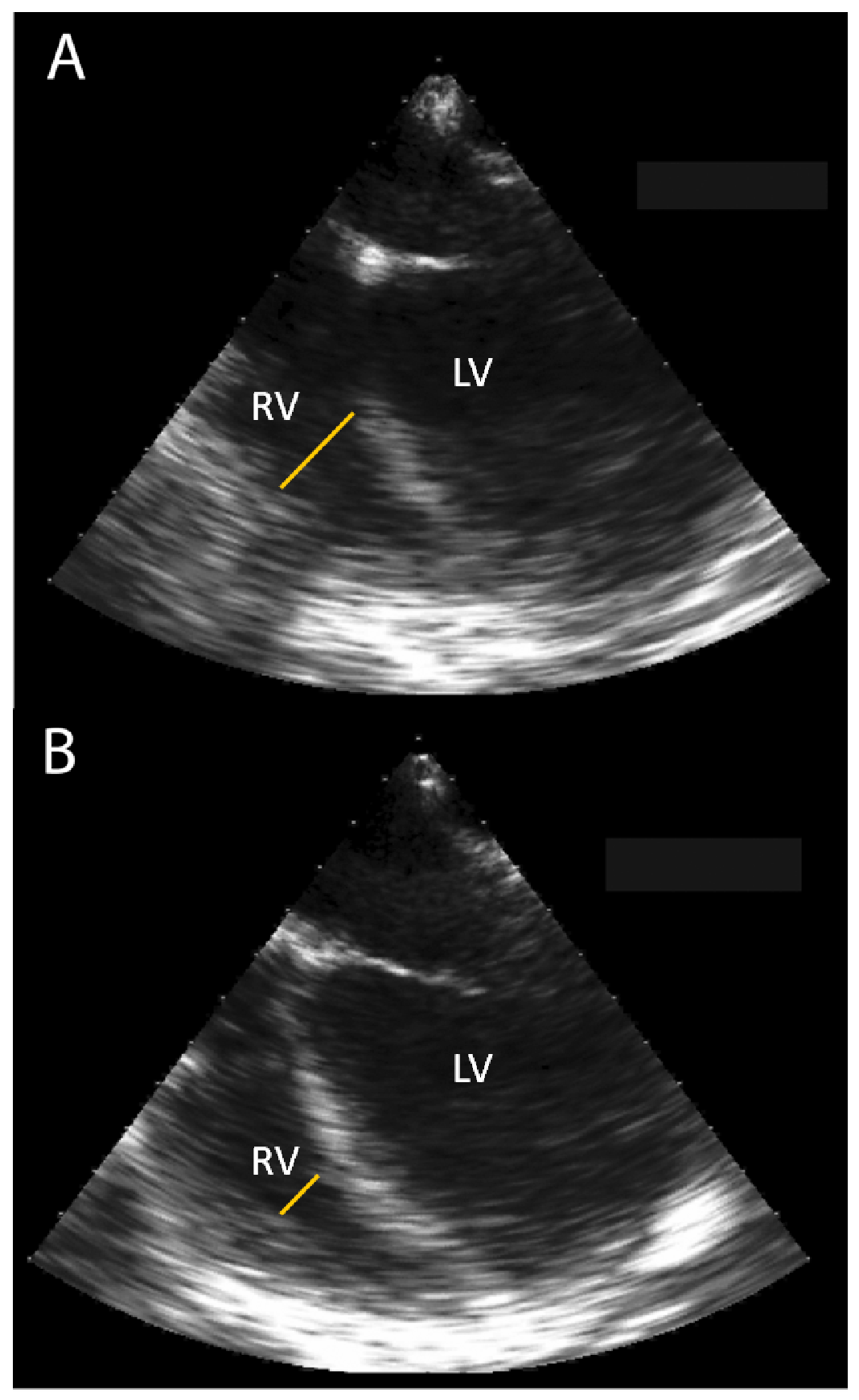


Figure 2. A. hTEE shows no cardiac tamponade. B. Cardiac standstill initiated immediate ECMO support. LV: left ventricle; RV: right ventricle.

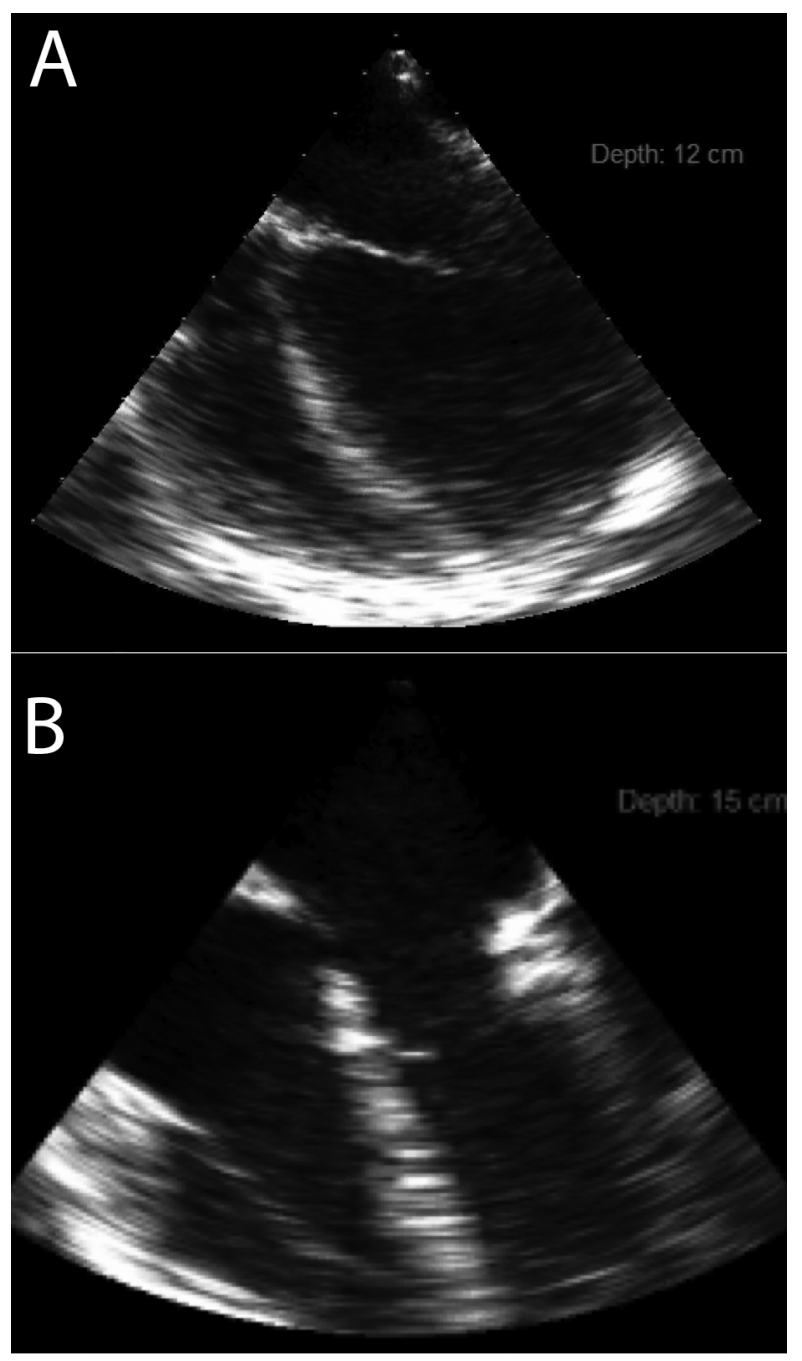


Figure 3. A. Transesophageal echocardiography with hTEE probe reveals thrombus (indicated by arrow) appearing to block ECMO venous cannula in right atrium (indicated by ellipse, cannula visible as parallel lines) - a possible cause for decreased flow (2 L/min), the presumed diagnosis after hTEE examination. B. Adjustment of cannula (indicated by ellipse, cannula visible as parallel lines) and ECMO RPM displaced and released the thrombus (indicated by arrow) from the catheter opening - this was further confirmed by resumption of ECMO flow at $4.5 \mathrm{~L} / \mathrm{min}$.

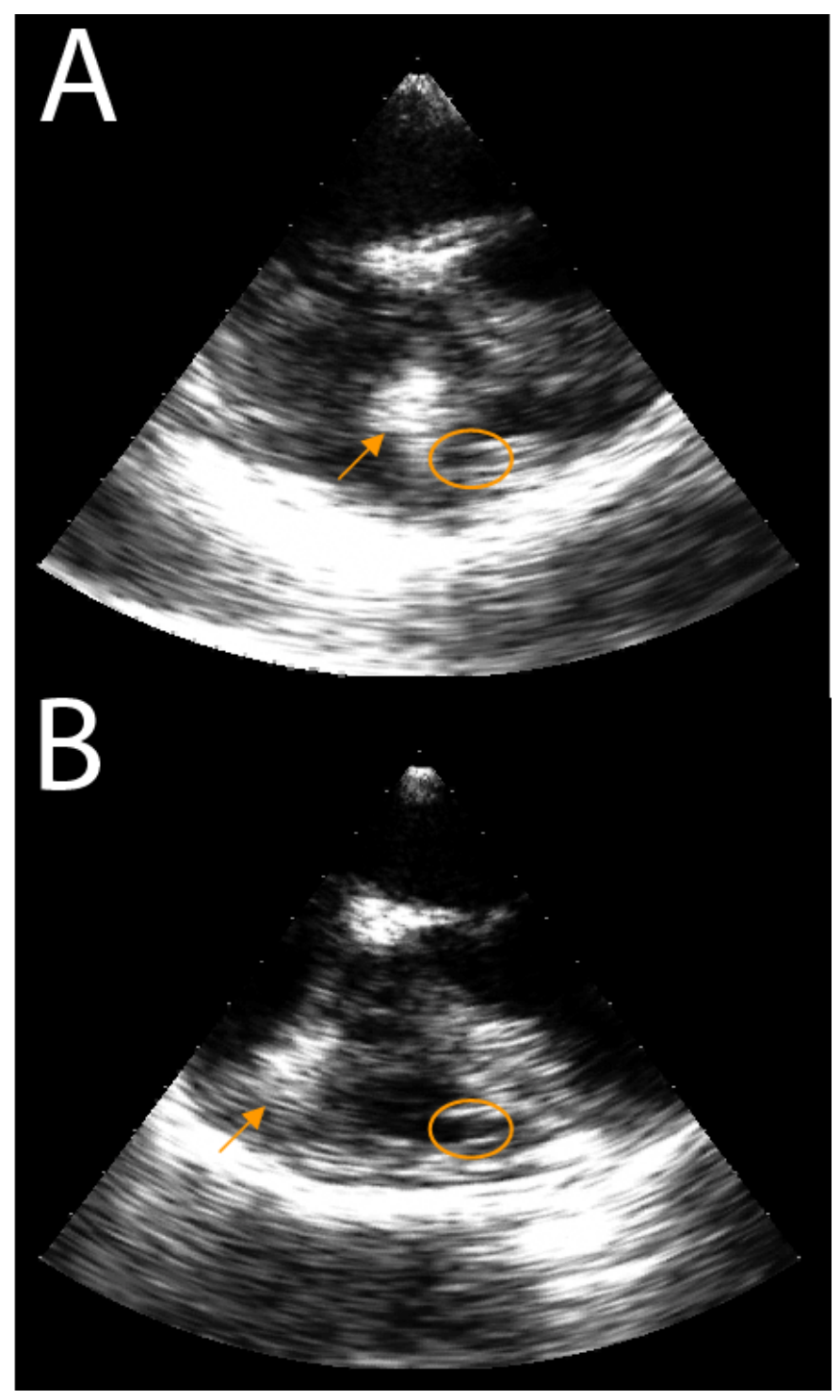

